University of Szeged

\title{
Comparison of Adequacy of Anaesthesia Monitoring with Standard Clinical Practice during routine general anaesthesia: An International, Multi-Centre, Single-blinded Randomized Controlled Trial.
}

\author{
M. Gruenewald ${ }^{1 \#}$, J. Harju²\#, B. Preckel ${ }^{3}$, Z. Molnár ${ }^{4}$, A. Yli-Hankala ${ }^{5}$, B. Bein ${ }^{6}$, \\ The AoA study group
}

\begin{abstract}
1 University Hospital Schleswig-Holstein - Kiel (Germany), ${ }^{2}$ Tampere University Hospital - Tampere (Finland), ${ }^{3}$ University of Amsterdam - Amsterdam (Netherlands), ${ }^{4}$ University of Szeged - Szeged (Hungary), ${ }^{5}$ Tampere University Hospital - Tampere (Hungary), ${ }^{6}$ Asklepios Hospital St. Georg - Hamburg (Germany), ${ }^{\#}$ contributed equally
\end{abstract}

\section{Background and Goal of Study:}

The use of clinical signs such as hypertension or tachycardia for guidance of anaesthesia can be challenging. Previous studies have suggested that incidence of unwanted anaesthesia events could be reduced by measuring the components of anaesthesia i.e. hypnosis and analgesia $(1,2)$. However, the evidence regarding the benefits for such monitoring is still limited (3).

\section{Materials and Methods:}

After approval of each ethics committee, a total of 494 patients were recruited in a multi-centre, single-blinded, randomized controlled trial (RCT). Patients were randomized into two groups and anaesthesia was guided with Entropy (hypnosis) and surgical pleth index SPI (analgesia) in (adequacy of anaesthesia group; AoA group) or standard monitoring alone (Control group). Anaesthesia was conducted with $\mathrm{TCl}$ of propofol and remifentanil. The patients were followed for signs of inadequate anaesthesia (i.e. grimacing, hypertension or tachycardia) or signs of too deep anaesthesia (i.e. hypotension and bradycardia) namely unwanted anaesthesia events.

\section{Table 1:}

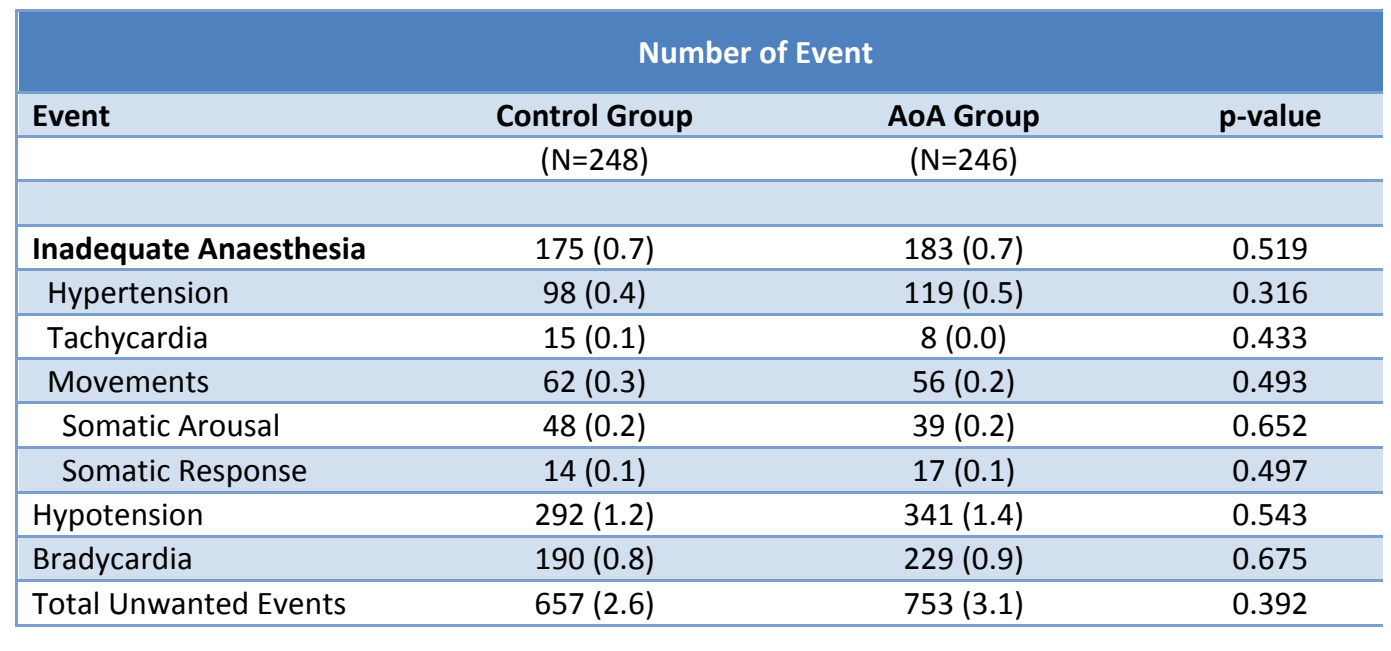

Figure 1:

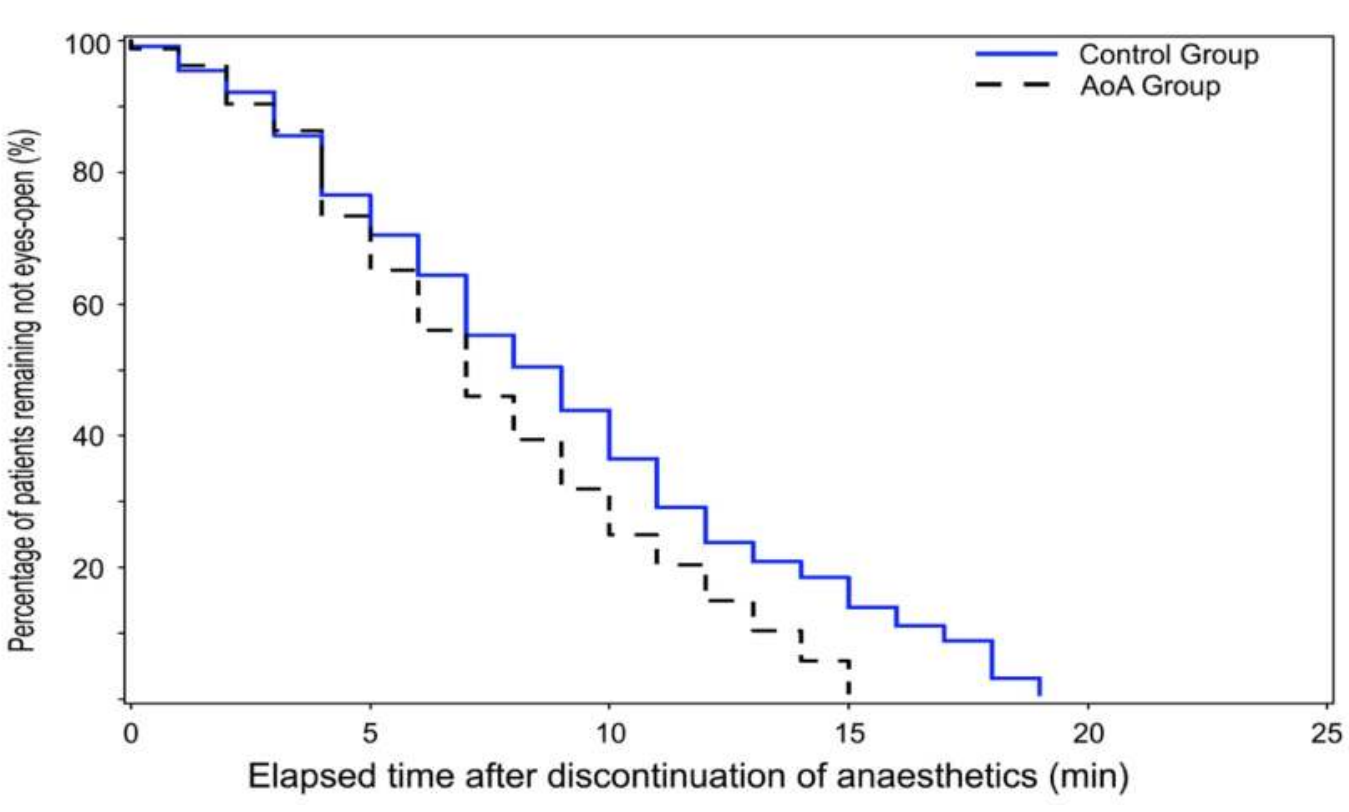

Results and Discussion:

Incidence of unwanted events was 0.7 events per subject in both groups ( $p=0.519$; Table 1$)$. In the AoA group, the overall consumption of propofol was significantly reduced with 6.88 vs. $7.53 \mathrm{mg} / \mathrm{kg} / \mathrm{h}$ in Control group ( $p<0.01$ ). Time of emergence was significantly shorter in AoA group with 8.0 vs. 9.6 min in Control group ( $p<0.01$; Figure 1). With respect to post-anaesthesia care, we found a trend towards faster full recovery with AoA monitoring but no difference with respect to pain or pain medications.

\section{Conclusion:}

In the present RCT, the guidance of TIVA by Entropy and SPI in comparison to standard monitoring alone was unable to reduce unwanted events. However, there was a slight reduction in the use of propofol and shorter times for emergence. The findings may have been influenced by low-risk profile of surgeries with low frequency of unwanted events. 